\title{
Pericias psicológicas y otros medios probatorios en las decisiones en las comisarías de familia de Bogotá: casos de violencia de pareja contra la mujer
}

\section{Forensic Psychological Evaluation and Other Evidences to the Decision Making Process in Administrative Family Courts in Bogotá: Partner Violence Against Women}

\author{
Martha Stella Ospino Rodríguez ${ }^{* *}$ \\ Universidad Santo Tomás \\ Universidad Cooperativa de Colombia - \\ Neiva \\ Claudia Victoria Vidal Padilla \\ Universidad Santo Tomás \\ Olga Lucía Valencia, \\ Universidad Santo Tomás \\ Universidad Complutense de Madrid \\ Raúl Oyuela-Vargas, \\ Universidad Santo Tomás \\ Recibido: 22 de mayo de 2011 \\ Revisado: 7 de julio de 2011 \\ Aceptado: 13 de octubre de 2011
}

\section{Resumen}

En este artículo se presenta el estudio que establece la asociación de las pericias psicológicas y otros medios probatorios con las decisiones de los operadores de justicia en las Comisarías de Familia de Bogotá; para lo cual, se realizó una investigación con diseño analítico de corte transversal descriptivo con 47 mujeres víctimas de violencia de pareja que solicitaron medida de protección en el Centro de Atención Integral contra la Violencia Intrafamiliar (CAVIF). Se analizaron los fallos de las medidas de protección definitivas impuestas en 16 comisarías de familia de 13 localidades de Bogotá. Los resultados mostraron que no existe asociación entre la utilización de las pericias psicológicas, dictamen de lesiones no fatales del Instituto Nacional de Medicina Legal y Ciencias Forenses (INMLCF), informes sociales, denuncias de Fiscalía y testimonios de terceros, con las decisiones tomadas por los comisarios de familia. Solo se encontró asociación entre la aceptación de cargos,

* Artículo producto de investigación.

** Correspondencia: Martha Stella Ospino Rodríguez Universidad Santo Tomás y Universidad Cooperativa de Colombia sede Neiva. Correo electrónico: msospinor@gmail.com. Claudia Victoria Vidal Padilla. Universidad Santo Tomás. Correo: claudiavictoriavidal@gmail.com. 
con las decisiones de aprobación de acuerdo conciliatorio y conminación a no agresión del accionado.

Palabras clave: violencia de pareja, evaluación psicológica forense, medios probatorios, medidas de protección, toma de decisiones judiciales.

\section{Abstract}

This study aimed to establish the association of the forensic psychological evaluation and other evidences to the decision making process by judicial operators in administrative family courts in Bogotá, through a research with a transversal descriptive cut analytical design on 47 women, victims of partner violence, who sought protective measures at the Integral Care Center against Domestic Violence (CAVIF). Analyzing the final protective measures imposed in 16 administrative family courts on Bogotá, the results showed no association between the use of forensic psychological evaluations, expert's concept of nonfatal injuries from the National Institute of Legal Medicine and Forensic Sciences (INMLCF), social forensic report, legal complaint, witness testimony and the decisions made by the family commissioner. The only association found was between acceptance of charges and approval decisions for settlement's agreements, as well as with the party's injunction to non-aggression.

Keywords: domestic violence, forensic psychological evaluation, evidentiary means, protective measures, judicial decision making.

\section{Introducción}

La violencia intrafamiliar se percibe como uno de los principales fenómenos sociales actuales, especialmente cuando se realizan seguimientos desde los medios de comunicación en casos concretos, en los cuales la violencia desencadena en lesiones significativas e incluso en la muerte, especialmente de mujeres a manos de sus compañeros, cónyuges o ex parejas. Las estadísticas de violencia de pareja están en aumento, como señala el Instituto Nacional de Medicina Legal y Ciencias Forenses, INMLCF, quien reportó que en el año 2006 se presentaron 43.319 casos; en el año 2007, 46.315 casos; en el 2008, 53.533 casos y, en el 2009, 61.008 casos, siendo las mujeres las principales víctimas (Instituto Nacional de Medicina Legal y Ciencias Forenses, 2006, 2007, 2008 y 2009). En este mismo sentido, se expresaron Profamilia (2005) y la Secretaría Distrital de Integración Social con sus reportes de violencia contra la mujer atendidos en las comisarías de familia de Bogotá (Secretaría Distrital de Integración Social, 2007a y 2007b) y la Procuraduría General de la Nación (2009), revelando las cifras de la Fiscalía.

De hecho, la violencia intrafamiliar marca un cambio en el imaginario de familia, en todos aquellos comportamientos esperados frente a la misma, como son: la protección, el cuidado, el crecimiento y la toma de decisiones conjuntas, dado que es definida en términos de los comportamientos realizados por uno o más de sus integrantes que causan daños o vulneran los derechos de los demás, tanto si se encuentran en convivencia como si tienen una relación vinculante. Las definiciones dadas por Alonso \& Castellanos (2006), Corsi, Domen \& Sotés (1995), Dutton \& Golant (1997), Echeburúa \& de Corral (1998), Ravazzola (1999) \& Rosen, Stith \& Williams (1992) resaltan dinámicas de poder al interior de la familia, donde una de las personas ejerce fuerza para tener dominio, control o superioridad sobre los otros integrantes; desequilibrio o desigualdad que suele presentarse por género, edad, condiciones culturales y recursos económicos, siendo las victimas más frecuentes mujeres, 
niños y niñas y adultos mayores. Por otro lado, Pérez, Lledias \& Giraldo (2008) se refieren a los "contextos familiares", como los escenarios de relaciones y procesos exógenos a la familia que están en permanente comunicación con ella, en una relación de doble vía. Dichos escenarios se crean y se recrean en la familia como procesos sociales, económicos, culturales e históricos.

El dominio del agresor, tal y como lo mencionan varios autores, se ejerce de diversas formas: a) físicamente mediante las acciones intencionales ejercidas sobre el cuerpo o sobre la salud de las personas que lo sufren (Echeburúa, 1994; Garzón, 2000; Labrador, Rincón, De Luis \& FernándezVelasco, 2004; Walker, 2000); siendo este tipo de violencia la más evidente y por ende a la que suele darse más importancia; b) emocional o psicológicamente en acciones de violencia verbal, lenguaje corporal de rechazo o descalificación, chantaje emocional (Echeburúa, 1994; Garzón, 2000; Labrador et ál., 2004, Ministerio de Salud, 1999, citado en Consejería Presidencial para la Política Social, 2001), siendo la violencia emocional o psicológica la "más difícil de identificar, en especial si se disfraza de "atención", o "preocupación", por la víctima, o si se consigue convencer a esta de que ella es la responsable del comportamiento del agresor" (Labrador et ál., 2004, p. 26); c) sexualmente, la imposición de actos de orden sexual, en los casos de pareja interpretado como obligación o deber, (Consejería Presidencial para la Política Social, 2001; Echeburúa, 1994, Labrador, et ál., 2004, Medina, 2002), y d) económica o patrimonialmente, como se resalta en la Ley 1257 de 2008.

La violencia en la pareja, señala la Organización Mundial de la Salud (2009) es "una de las formas más comunes de violencia contra la mujer" ( $p$. 1), con el agravante que presenta múltiples factores que se entrecruzan de manera circular para su génesis, mantenimiento y difícil erradicación, como lo señala la Consejería Presidencia para la Política Social (2001). Estos diversos factores son sociales como: la situación de violencia social, desigualdad, insatisfacción de necesidades básicas, predominio de condiciones de autoridad patriarcal, entre otros; factores familiares como: el inadecuado manejo de la ira, historia de violencia en la familia de origen, condiciones especiales de algún integrante del grupo (como por ejemplo una discapacidad), imposición de roles de la pareja y dependencia económica; e individuales, donde prevalece bajo nivel de autoestima, actitudes negativas hacia sí mismo, ansiedad, abuso de sustancias, dependencia, la subordinación a estereotipos, la normalización o legitimización de los significados de violencia, entre otros (Secretaría de Gobierno, 2003; Alonso \& Castellanos, 2006; Gómez, Ramos \& Rojas, 2010; Medina, 2002 Ravazzola, 1999; Schumacher, Feldbau, Smith Slep \& Heyman, 2001, citados en Cáceres, 2007).

Otro de los aspectos fundamentales a considerar son las consecuencias que se derivan de los eventos de violencia de pareja. Como señalan Cobo (1999) y el Instituto Nacional de Medicina Legal y Ciencias Forenses (2001) pueden ser físicas, como: fracturas, hematomas, cicatrices, limitaciones físicas, abortos, disfunciones sexuales, cefaleas, trastornos somatomorfos, hasta la muerte. Consecuencias emocionales, como la agresividad, irritabilidad, inseguridad, cogenerando o con causando trastornos de abuso o dependencia de sustancias, trastornos de estado de ánimo como depresión con riesgo suicida o autolesiones, trastornos de ansiedad como ataque de pánico, estrés postraumático, trastornos disociativos, trastornos adaptativos; y familiares: alteración de roles familiares y sociales (Amor, Echeburúa, De Corral, Zubizarreta \& Sarasua, 2002; Echeburúa, De Corral, Sarasua \& Zubizarreta, 1998; Jackson, Petritic-Jackson \& Witte, 2002; Pérez, 2002; Radford \& Hester, 2006; Sarasua, Zubizarreta, Echeburúa \& De Corral, 2007).

En los casos de violencia de pareja hacia la mujer, la afectación no solo se presenta en ella, es importante considerar también las consecuencias de la violencia en los hijos. Holden (2003) (citado en Atenciano, 2009) señala distintas formas de exposición de los niños a la violencia, que varían desde ser testigo directo, observar las consecuencias y las secuelas de la violencia en la madre, participar e intervenir, hasta ser víctimas de la misma, lo que genera que se presenten 
impactos diferenciados en ellos. La misma autora indica que:

Esta población presenta a corto y largo plazo dificultades emocionales y conductuales, y síntomas de trauma, asociados a los malos tratos contra sus madres, ejercidos durante la relación de pareja y tras la finalización de la misma. Además, un porcentaje elevado de estos menores sufre también maltrato físico, psicológico y sexual (p. 261).

Dentro de las dificultades frecuentes, Holden (2003, citado en Atenciano, 2009) menciona: ansiedad, depresión, baja autoestima, agresividad y problemas de conducta, baja competencia social, aprendizaje de la violencia como legítima, indicios de dificultades en la génesis de las relaciones de apego, problemas de conflictividad en la escuela, bajo rendimiento escolar, violencia hacia los pares, violencia hacia la progenitora, entre otros. Así mismo, Almonacid, Daroch y Mena (2006) señalan que "la violencia hacia los niños aumenta cuando existe violencia conyugal y un niño que ha sido golpeado cuando pequeño por sus padres, tiene una mayor probabilidad de ser un hombre golpeador que uno que no sido golpeado" (p. 6). Más aún, Busch, Zagar, Hughes, Arbit \& Bussell (1990, citados en Esbec y Gómez-Jarabo, 1999) afirman que, la historia de violencia intrafamiliar es un indicador de delincuencia violenta (homicidio) en la juventud.

En Colombia, una de las primeras y principales instancias para la atención de violencia de pareja es la Comisaría de Familia, cuya misión es "prevenir, garantizar, restablecer y reparar los derechos de los miembros de la familia conculcados por situaciones de violencia intrafamiliar y las demás establecidas por la ley" (Artículo 83, Ley 1098 del 2006). Las instituciones jurídicas al igual que:

(...) el marco jurídico también se pueden considerar como un elemento de presión social que sustenta prejuicios sobre los papeles formalizados de los miembros familiares, protegiendo la familia como una institución que se debe preservar a pesar de los efectos y manifestaciones que los conflictos generan sobre los individuos (Pérez, Lledias \& Giraldo, 2008, p.435).

Pero para poder conocer lo que sucede en la violencia intrafamiliar, los comisarios deben atender a la actividad probatoria, de manera que acuden como señala Arenas (2003) a los "recursos de conocimiento denominados medios de prueba. Estos son entre otros, el testimonio, el peritaje, el documento, la confesión...” (p. 110). En efecto, el Código de Procedimiento Civil se refiere a los medios de prueba afirmando que "sirven como pruebas, la declaración de parte, el juramento, el testimonio de terceros, el dictamen pericial, la inspección judicial, los documentos, los indicios y cualesquiera otros medios que sean útiles para la formación del convencimiento del juez" (Artículo 175). Por su parte, la Ley 575 de 2000 , señala las medidas de protección que se pueden establecer para detener y sancionar los eventos de violencia en la familia como son: ordenar al agresor abstenerse de realizar la conducta objeto de la queja; ordenar al agresor el desalojo de la casa de habitación que comparte con la víctima; ordenar al agresor abstenerse de penetrar en cualquier lugar donde se encuentre la víctima; prohibir al agresor esconder o trasladar de la residencia a los niños y personas discapacitadas miembros del grupo familiar; obligar a acudir a un tratamiento reeducativo y terapéutico; ordenar al agresor el pago de los gastos médicos, psicológicos y psíquicos que requiera la víctima; ordenar una protección temporal especial de la víctima por parte de las autoridades de policía; y cualquier otra medida necesaria para el cumplimiento de los propósitos de la dicha ley. Así mismo, la Ley 1257 de 2008 adicionó otras medidas de protección a las señaladas, tales como: suspender al agresor la tenencia, porte y uso de armas; decidir provisionalmente el régimen de visitas, la guarda y custodia de los hijos; decidir provisionalmente quién tendrá a su cargo las pensiones alimentarias; decidir provisionalmente el uso y disfrute de la vivienda familiar; prohibir al agresor la realización de cualquier acto de enajenación o gravamen de bienes de su propiedad, sujetos a registro si tuviere sociedad conyugal o patrimonial vigente; ordenar al agresor la devolución inmediata de los objetos de uso personal, documentos de identidad y cualquier 
otro documento u objeto de propiedad o custodia de la víctima.

Con base en lo anterior, acerca del incremento de la violencia de pareja hacia la mujer en los últimos años en Colombia, la afectación y consecuencias de la misma en las mujeres y los hijos, de la necesidad del análisis de los medios probatorios frente a la toma de decisiones a través de medidas de protección en instancias como las comisarías de familia, surge entonces el interés por conocer cómo las pericias psicológicas y otros medios probatorios son pertinentes en las actuaciones procesales, los aportes que el desarrollo de los mismos pueden generar sobre medidas concretas para la intervención de las familias no solo en términos de la sanción de la violencia sino a su prevención y a la reparación a la víctima, y las necesidades de ajustes en los equipos profesionales en las entidades que atienden esta problemática. Específicamente, el objetivo del presente estudio es establecer la asociación de las pericias psicológicas y otros medios probatorios con las decisiones de los comisarios de familia en la medida de protección por violencia de pareja contra las mujeres.

\section{Método}

Esta investigación se desarrolló a través de un diseño no experimental analítico de corte transversal descriptivo. Es transversal en tanto recolectó la información en un periodo de tiempo dado (Ospino, 2004), entre febrero y abril del 2010. En el estudio participaron 47 mujeres víctimas de violencia de pareja que formularon denuncia en la Comisaría de Familia del Centro de Atención Integral Contra la Violencia intrafamiliar $(\mathrm{CAVIF})^{1}$ en el mencionado período, las cuales se encontraron distribuidas de la siguiente manera: a 13 mujeres se les realizó una pericia psicológi$\mathrm{ca}$, dado que su reporte señalaba violencia verbal o psicológica sin agresiones físicas recientes, se seleccionaron 24 mujeres a quienes el Instituto

$1 \quad$ El CAVIF fue creado en coordinación institucional con 11 entidades del Distrito y la Fiscalía General de la Nación para "restaurar familias en situación de violencia intrafamiliar y sexual, generando una sinergia entre la infraestructura de justicia ya creada y [el] CAVIF" (Fiscalía General de la Nación, Acuerdo 123 de 2004, citado en Rodríguez, Padilla, Rodríguez \& Díaz, 2010).
Nacional de Medicina Legal y Ciencias Forenses (INMLCF) había realizado dictámenes de lesiones médico legales porque su relato indicaba la presencia de violencia física reciente, y a 10 mujeres que contaban con dictamen de lesiones no fatales del INMLCF, por expresar que habían sido agredidas físicamente, se les realizó adicionalmente la pericia psicológica. En relación con el procedimiento, se efectúo el análisis a través de una matriz en la que se registraron los resultados de cada uno de los casos, tanto de los medios de prueba utilizados como de las diferentes decisiones establecidas por los comisarios de familia, en las medidas de protección definitivas y los datos obtenidos en las pericias psicológicas y en los fallos que sirvieron para la presentación de los resultados y su discusión. La investigación se realizó en cinco fases: 1) Definición del protocolo de evaluación para la realización de las pericias; 2) Realización de las pericias psicológicas e identificación de casos; 3) Diseño de matriz; 4) Recolección de fallos de las medidas de protección y, 5) Análisis de resultados.

\section{Resultados}

Con la revisión de los expedientes de las medidas de protección definitivas tomadas en las comisarías de familia, se realizó el análisis descriptivo de la variable medios probatorios, en donde se encontraron los resultados observados en la Tabla 1. El $72 \%$ de los casos remitidos por el CAVIF a las comisarías de familia (en adelante CF), correspondientes por jurisdicción territorial contaban con dictamen de lesiones no fatales del INMLCF para las medidas de protección; los comisarios utilizaron este dictamen como medio probatorio en el $82 \%$ de los casos en las medidas de protección definitivas. El $49 \%$ de los expedientes remitidos por el CAVIF tenían pericia psicológica, y en ninguna CF de la localidad se ordenó la práctica de esta para los casos en que la medida de protección provisional no contara con ella, por tanto, el porcentaje de utilización de la pericia psicológica es del $28 \%$ como aparece en la Tabla 1 . En todos los expedientes la víctima de violencia intrafamiliar diligenció una solicitud de medida de protección en la que consignó su testimonio respecto a los 
hechos de violencia y fue tenido en cuenta en su totalidad para dictar la medida de protección definitiva en las CF de la localidad. Igualmente, desde el CAVIF se remitieron el $100 \%$ de las medidas de protección provisionales con la denuncia a Fiscalía por el delito de violencia intrafamiliar por parte de la accionante y esta fue usada como medio probatorio en el $45 \%$ de los casos. En cuanto a otros medios probatorios, se observa que en los casos en que existía la posibilidad de aceptación de cargos por parte del accionado dado que este compareció a la audiencia (66\% de casos), dicho reconocimiento o aceptación se dio en el $87 \%$ de ellos. En las audiencias en las cuales no compareció el accionado, a pesar de haber sido debidamente notificado (34\%), se utilizó el medio probatorio aceptación de cargos por no asistencia por parte del accionado en el $100 \%$ de los casos.
Finalmente, en las CF de la localidad tan solo se solicitó, como medio probatorio, el Informe social y el testimonio a terceros en un $2 \%$ para cada uno de estos. Los resultados de la utilización para dictar la medida de protección en las CF de la localidad, de los medios probatorios dictamen de lesiones no fatales del INMLCF o pericias psicológicas en los casos en que estos medios probatorios se encontraban en el expediente debido a la remisión de los mismos desde el CAVIF y se hallaban de manera exclusiva, es decir, que no se presentaban los dos simultáneamente, fueron de $82 \%$ y de $62 \%$, respectivamente. En aquellos casos en los cuales estos dos medios de prueba se encontraban simultáneamente, el porcentaje de utilización en la CF de la localidad fue de $80 \%$ para el dictamen de lesiones no fatales del INMLCF y de $50 \%$ para las pericias psicológicas.

Tabla 1.

Frecuencias y porcentajes de utilización de medios probatorios en los procesos de medidas de protección definitivas por violencia de pareja contra las mujeres en las comisarías de familia de Bogotá

\begin{tabular}{lccccccccc}
\hline & \multicolumn{2}{c}{ NO } & \multicolumn{2}{c}{ sí } & \multicolumn{2}{c}{ NO APLICA } & \multicolumn{2}{c}{ TOTAL } \\
\hline Medios probatorios & $\mathrm{f}$ & $\%$ & $\mathrm{f}$ & $\%$ & $\mathrm{f}$ & $\%$ & $\mathrm{~F}$ & $\%$ \\
\hline Dictamen lesiones de INMLCF & 6 & 13 & 28 & 59 & 13 & 28 & 47 & 100 \\
Pericia psicológica & 34 & 72 & 13 & 28 & 0 & 0 & 47 & 100 \\
Testimonio de la accionante & 0 & 0 & 47 & 100 & 0 & 0 & 47 & 100 \\
Aceptación de cargos accionado & 4 & 9 & 27 & 57 & 16 & 34 & 47 & 100 \\
Aceptación cargos por no asistencia & 0 & 0 & 16 & 34 & 31 & 66 & 47 & 100 \\
Denuncia en Fiscalía & 26 & 55 & 21 & 45 & 0 & 0 & 47 & 100 \\
Informe social & 46 & 98 & 1 & 2 & 0 & 0 & 47 & 100 \\
Testimonio de terceros & 46 & 98 & 1 & 2 & 0 & 0 & 47 & 100 \\
\hline
\end{tabular}

Posteriormente, se analizaron los resultados pertinentes obtenidos en las pericias psicológicas para la toma de decisiones por los operadores de justicia en las CF, como son: el comportamiento violento en presencia de los hijos, que se reporta en el $87 \%$; la utilización de arma blanca o de fuego para la intimidación o amenaza en el $13 \%$; el consumo de alcohol en el $52 \%$ o el consumo de spa en el 13\%; los resultados de las pruebas psico- lógicas que indican que se presenta depresión en el $74 \%$, ansiedad en el $83 \%$, baja autoestima en el $35 \%$, trastorno de estrés postraumático en el $39 \%$.

Igualmente, se realizó el análisis descriptivo de la variable decisiones tomadas por los/as comisarios/as en las medidas de protección definitivas en las CF, cuyos resultados pueden apreciarse en la Tabla 2. 
Tabla 2.

Frecuencias y porcentajes de las decisiones tomadas en los procesos de medidas de protección definitivas por violencia de pareja contra las mujeres en las comisarías de familia de Bogotá

\begin{tabular}{|c|c|c|c|c|c|c|c|c|}
\hline \multirow[t]{2}{*}{ Decisiones } & \multicolumn{2}{|c|}{ NO } & \multicolumn{2}{|c|}{ sí } & \multicolumn{2}{|c|}{ NO APLICA } & \multicolumn{2}{|c|}{ TOTAL } \\
\hline & $\mathbf{f}$ & $\%$ & $\mathrm{f}$ & $\%$ & 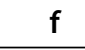 & $\%$ & $\mathbf{F}$ & $\%$ \\
\hline Aprobación acuerdo conciliatorio & 8 & 17 & 24 & 51 & 15 & 32 & 47 & 100 \\
\hline Conminación no agresión accionado & 8 & 17 & 39 & 83 & 0 & 0 & 47 & 100 \\
\hline Conminación no agresión a partes & 43 & 91 & 4 & 9 & 0 & 0 & 47 & 100 \\
\hline Medida protección hijos & 26 & 55 & 21 & 45 & 0 & 0 & 47 & 100 \\
\hline Desalojo accionado & 45 & 96 & 2 & 4 & 0 & 0 & 47 & 100 \\
\hline Abstenerse de ingreso & 41 & 87 & 6 & 13 & 0 & 0 & 47 & 100 \\
\hline Apoyo policivo & 36 & 77 & 11 & 23 & 0 & 0 & 47 & 100 \\
\hline No intimidar con armas & 45 & 96 & 2 & 4 & 0 & 0 & 47 & 100 \\
\hline Tratamiento al accionado & 39 & 83 & 8 & 17 & 0 & 0 & 47 & 100 \\
\hline Tratamiento a las partes & 12 & 25 & 35 & 75 & 0 & 0 & 47 & 100 \\
\hline Tratamiento específico accionado & 43 & 91 & 4 & 9 & 0 & 0 & 47 & 100 \\
\hline Pago gastos & 46 & 98 & 1 & 2 & 0 & 0 & 47 & 100 \\
\hline No ingreso con alcohol/spa & 45 & 96 & 2 & 4 & 0 & 0 & 47 & 100 \\
\hline Taller pautas crianza & 36 & 77 & 11 & 23 & 0 & 0 & 47 & 100 \\
\hline Seguimiento & 1 & 2 & 46 & 98 & 0 & 0 & 47 & 100 \\
\hline Otras medidas de protección & 40 & 85 & 7 & 15 & 0 & 0 & 47 & 100 \\
\hline
\end{tabular}

Por último, los datos fueron analizados mediante las medidas de asociación: coeficiente de Phi para las variables dicotómicas y coeficiente $\mathrm{V}$ de
Cramer para las politómicas con un $a=0.05$. Los resultados de las asociaciones se presentan en las tablas 3 y 4 .

Tabla 3.

Coeficientes de asociación Phi de medios probatorios y decisiones tomadas en los procesos de medidas de protección definitivas por violencia de pareja contra las mujeres en las comisarías de familia de Bogotá

\begin{tabular}{|c|c|c|c|c|c|c|c|c|}
\hline \multirow[t]{2}{*}{ Decisiones } & \multicolumn{2}{|c|}{ Pericia Psicológica } & \multicolumn{2}{|c|}{$\begin{array}{l}\text { Denuncia } \\
\text { Fiscalía }\end{array}$} & \multicolumn{2}{|c|}{ Informe social } & \multicolumn{2}{|c|}{$\begin{array}{l}\text { Testimonio } \\
\text { terceros }\end{array}$} \\
\hline & Phi & $\begin{array}{c}\text { Sig } \\
\text { aprox }\end{array}$ & Phi & $\begin{array}{l}\text { Sig } \\
\text { aprox }\end{array}$ & Phi & $\begin{array}{c}\text { Sig } \\
\text { aprox }\end{array}$ & Phi & $\begin{array}{c}\text { Sig } \\
\text { aprox }\end{array}$ \\
\hline Aprobación acuerdo conciliatorio & .214 & .342 & .399 & .024 & .326 & .083 & .215 & .336 \\
\hline Conminación no agresión accionado & .027 & .854 & .179 & .219 & .067 & .647 & .067 & .647 \\
\hline Tratamiento al accionado & -.089 & .542 & .160 & .272 & -.077 & .599 & -.077 & .599 \\
\hline Tratamiento a las partes & .035 & .811 & -.061 & .270 & -.256 & .084 & .086 & .554 \\
\hline Desalojo accionado & .105 & .470 & .023 & .877 & -.031 & .831 & -.031 & .831 \\
\hline Abstenerse de ingreso & .049 & .739 & -.169 & .246 & -.056 & .699 & -.056 & .699 \\
\hline Pago gastos & -.091 & .532 & -.133 & .364 & -.022 & .882 & -.022 & .882 \\
\hline Apoyo policivo & -.005 & .974 & .211 & .148 & .267 & .067 & .267 & .067 \\
\hline Seguimiento & .091 & .532 & -.164 & .261 & 0.22 & .882 & 0.22 & .882 \\
\hline Medida protección hijos & -.077 & .596 & -.033 & .821 & .164 & .261 & -.133 & .364 \\
\hline
\end{tabular}




\begin{tabular}{lcccccccc}
\hline \multicolumn{1}{c}{ Decisiones } & \multicolumn{2}{c}{ Pericia Psicológica } & \multicolumn{2}{c}{$\begin{array}{c}\text { Denuncia } \\
\text { Fiscalía }\end{array}$} & \multicolumn{2}{c}{ Informe social } & \multicolumn{2}{c}{$\begin{array}{c}\text { Testimonio } \\
\text { terceros }\end{array}$} \\
\hline & Phi & $\begin{array}{c}\text { Sig } \\
\text { aprox }\end{array}$ & Phi & $\begin{array}{c}\text { Sig } \\
\text { aprox }\end{array}$ & Phi & $\begin{array}{c}\text { Sig } \\
\text { aprox }\end{array}$ & Phi & $\begin{array}{c}\text { Sig } \\
\text { aprox }\end{array}$ \\
\hline Conminación no agresión a partes & -.018 & .901 & -.274 & .060 & -.045 & .758 & -.045 & .758 \\
Tratamiento específico accionado & .152 & .296 & .033 & .823 & -.045 & .758 & -.045 & .758 \\
No intimidar con armas & -.130 & .371 & .235 & .108 & -.031 & .831 & -.031 & .831 \\
No ingreso con alcohol/spa & .105 & .470 & .235 & .108 & -.031 & .831 & -.031 & .831 \\
Taller pautas crianza & -.117 & .422 & .009 & .953 & -.082 & .576 & .267 & .067 \\
Otras medidas de protección & .259 & .534 & .310 & .340 & .062 & .996 & .062 & 0996 \\
\hline
\end{tabular}

Como se observa en la Tabla 3, no fueron estadísticamente significativas las asociaciones realizadas con el Coeficiente Phi para los medios proba- torios: pericia psicológica, denuncia en Fiscalía, informe social y testimonio de terceros con ninguna de las decisiones tomadas en las comisarías.

Tabla 4.

Coeficientes $V$ de Cramer de medios probatorios y decisiones tomadas en los procesos de medidas de protección definitivas por violencia de pareja contra las mujeres en las comisarías de familia de Bogotá

\begin{tabular}{lcccccccc}
\hline \multicolumn{1}{c}{ Decisiones } & \multicolumn{2}{c}{$\begin{array}{c}\text { Pericias } \\
\text { psicológicas }\end{array}$} & \multicolumn{2}{c}{$\begin{array}{c}\text { Dictamen } \\
\text { INMLCF }\end{array}$} & \multicolumn{2}{c}{$\begin{array}{c}\text { Aceptación en } \\
\text { audiencia }\end{array}$} & \multicolumn{2}{c}{$\begin{array}{c}\text { Aceptación no } \\
\text { asiste }\end{array}$} \\
\cline { 2 - 10 } & $\begin{array}{c}\text { V } \\
\text { Cram }\end{array}$ & $\begin{array}{c}\text { Sig } \\
\text { aprox }\end{array}$ & V Cram & $\begin{array}{c}\text { Sig } \\
\text { aprox }\end{array}$ & V Cram & $\begin{array}{c}\text { Sig } \\
\text { aprox }\end{array}$ & $\begin{array}{c}\text { V } \\
\text { Cram }\end{array}$ & $\begin{array}{c}\text { Sig } \\
\text { aprox }\end{array}$ \\
\hline Aprobación acuerdo conciliatorio & .214 & .342 & .227 & .302 & .698 & .000 & .958 & .000 \\
Conminación no agresión accionado & .027 & .854 & .383 & .032 & .523 & .002 & .325 & .026 \\
Tratamiento al accionado & .089 & .542 & .047 & .950 & .214 & .341 & .175 & .230 \\
Tratamiento a las partes & .035 & .811 & .097 & .803 & .201 & .386 & .197 & .176 \\
Desalojo accionado & .105 & .470 & .120 & .713 & .181 & .461 & .151 & .299 \\
Abstenerse de ingreso & .049 & .739 & .147 & .602 & .270 & .180 & .263 & .071 \\
Pago gastos & .091 & .532 & .121 & .707 & .483 & .004 & .106 & .468 \\
Apoyo policivo & .005 & .974 & .220 & .322 & .357 & .050 & .345 & .018 \\
Seguimiento & .091 & .532 & .385 & .030 & .127 & .685 & .106 & .468 \\
Medida protección hijos & .077 & .596 & .225 & .304 & .127 & .685 & .013 & .927 \\
Conminación no agresión a partes & .018 & .901 & .173 & .493 & .470 & .006 & .219 & .133 \\
Tratamiento específico & .152 & .296 & .173 & .493 & .126 & .690 & .103 & .481 \\
accionado & & & & & & & & \\
No intimidar con armas & .130 & .371 & .174 & .492 & .087 & .838 & .071 & .626 \\
No ingreso con alcohol/spa & .105 & .470 & .120 & .713 & .293 & .132 & .293 & .044 \\
Taller pautas crianza & .117 & .422 & .220 & .320 & .206 & .368 & .079 & .588 \\
Otras medidas de protección & .259 & .534 & .227 & .302 & .218 & .814 & .254 & .554 \\
\hline
\end{tabular}

Fueron estadísticamente significativas las asociaciones realizadas con el Coeficiente $V$ de Cramer para el medio probatorio: aceptación de cargos por parte del accionado; con las decisiones: aprobación de acuerdo conciliatorio y conminación al accionado a no agresión, como puede apreciarse en la Tabla 4. Pero no se encontraron asociaciones estadísticamente significativas de este medio con las demás decisiones. 


\section{Discusión y conclusiones}

De los resultados obtenidos en la presente investigación, se puede observar que los medios probatorios más utilizados por los comisarios son: el testimonio por parte de la accionante y la aceptación de cargos por no asistencia de parte del accionado, con un $100 \%$ para cada uno de ellos, resultados que se esperaban en tanto que la Ley 575 de 2000 que modifica la Ley 294 de 1996, establece como procedimiento que "la petición de medida de protección podrá formularse por escrito..." (Artículo 5). Adicionalmente, esta misma ley dice que "si el agresor no compareciere a la audiencia se entenderá que acepta los cargos formulados en su contra" (Artículo 9), lo que indica que estos medios probatorios se convierten en elementos fundamentales en la toma de decisiones de los comisarios de familia.

En segundo lugar de relevancia, se encuentra la aceptación de cargos por el accionado durante la audiencia de comparecencia, lo que indica que los agresores hacen un reconocimiento de su violencia contra la mujer en un $87 \%$ de los casos. Como señala Arenas (2003), la confesión puede ser interpretada de diferentes maneras por el operador de justicia que determina su validez; en la presente investigación se observa que, en todos los casos en los cuales el accionado señaló que había generado acciones de violencia de pareja dentro de los descargos que rindió, esta se tuvo como medio de prueba. Este es el único medio probatorio que se encontró asociado con dos de las decisiones tomadas por los comisarios: aprobación de acuerdo conciliatorio y conminación a no agresión del accionado, de acuerdo con el análisis estadístico realizado por medio del Coeficiente V de Cramer (Tabla 4).

El tercer medio probatorio de mayor utilización es el dictamen técnico médico legal de lesiones no fatales, con el $82 \%$; en cuarto lugar, se halla la pericia psicológica con una utilización del $57 \%$, cuando se analiza teniendo en cuenta las pericias disponibles por la remisión desde el CAVIF; mientras que, cuando se toma la totalidad de los casos debido a la posibilidad de realizarla en todos los procesos a solicitud de las partes o de manera oficiosa a solicitud del Despacho, se observa que su utilización y, por tanto, su relevancia podría ubicarse en el quinto lugar con $28 \%$ como se observa en la Tabla 1, con un nivel de importancia bajo, confirmado por la medida de asociación Coeficiente Phi, que reveló que no existe asociación entre la utilización de la pericia psicológica y las decisiones tomadas en los procesos de protección definitivos (Tabla 3).

En quinto lugar, se encuentra la denuncia por parte de la accionante en la Fiscalía por el delito de violencia intrafamiliar con un $45 \%$, a pesar que existía copia de la denuncia formulada en la Fiscalía de CAVIF, en la totalidad de las medidas de protección analizadas en el presente estudio, por el principio de la sana crítica (Artículo 187 del Código de Procedimiento Civil-CPC), los comisarios de familia apreciaron las pruebas en conjunto y decidieron su pertinencia y eficacia para el proceso de violencia intrafamiliar de manera diferencial. Finalmente, los medios probatorios menos utilizados por los comisarios son: el Informe social y el testimonio a terceros, con un $2 \%$ de utilización.

Según el Artículo 175 del CPC, los dictámenes periciales son medios probatorios empleados "para la formación del convencimiento del juez". En las CF, como se pudo apreciar en los resultados, se utilizaron los dictámenes periciales médico legales, las pericias psicológicas realizadas por una de las investigadoras en el CAVIF y los informes sociales realizados por los trabajadores sociales de los equipos profesionales de las CF de la localidad. Dado que tan solo se utilizó en un $2 \%$ el Informe social, se analizó el comportamiento de las dos primeras periciales, encontrando que, en los casos en que los comisarios de familia contaban con las dos pericias simultáneamente, en las localidades hicieron una mayor utilización para la toma de decisiones en las medidas de protección definitivas del primero $(80 \%)$ frente a la pericia psicológica (50\%). De igual forma, se mantiene la misma tendencia cuando se analizaron los resultados en los casos en que solo se contaba con una de las pericias mencionadas, para el caso del 
dictamen de lesiones no fatales el $82 \%$ y para la pericia psicológica el $62 \%$.

Un argumento que se puede esgrimir a favor de no ordenar la realización de las pericias psicológicas en las $\mathrm{CF}$ es que no se requieren, partiendo del principio de la necesidad de la prueba (Artículo 175 del CPC). Esta sustentación tendría validez cuando se va únicamente a sancionar la ocurrencia, o no, de la violencia, la cual queda demostrada con la aceptación de los cargos por parte del accionado. Sin embargo, la violencia intrafamiliar es un fenómeno complejo que requiere unas medidas más amplias que la conminación a no agresión, tratamiento reeducativo y terapéutico, y el seguimiento a las medidas tomadas. Se podría concluir que los operadores de justicia de las CF no consideran de gran utilidad las pericias psicológicas para tomar las decisiones o a penas se está construyendo el reconocimiento de las mismas para tal fin. Esta hipótesis se mantiene con más fuerza cuando se analizan las dificultades encontradas para realizar la presente investigación: 1) Limitaciones para el acceso a la información por el estudio piloto realizado en siete $\mathrm{CF}, 2$ ) Trámites internos en las CF de las localidades que no permitieron la verificación de la información (dificultades para la revisión de expedientes aun cuando se contaba con la autorización para ello y la recepción de los casos en las CF o la ubicación de expedientes), 3) Desistimiento por parte de las víctimas o condiciones legales que conllevaron al cierre en las ordenes administrativas de medida de protección y, 4) La poca claridad acerca de la utilidad de las pericias psicológicas o de las solicitudes que se pueden realizar frente a las mismas, tanto en las CF de la localidad, como en el CAVIF mismo.

Dentro de los resultados esperados de las decisiones tomadas por los comisarios, en el presente estudio, está el establecimiento de sanciones debido a la violencia de pareja hacia la mujer, como se señala en la Ley 294 de 1996, modificada por la Ley 575 de 2000, se busca prevenir, remediar y sancionar la violencia, lo cual se confirmó parcialmente toda vez que las decisiones con mayor porcentaje fueron la conminación frente a la violencia (92\%, Tabla 2), y la obligación de asis- tir a tratamiento reeducativo y terapéutico $(92 \%$, Tabla 2); sin embargo, era de esperarse que en el $100 \%$ se realizara conminación; ya sea a alguna o ambas partes, en tanto que presentaron acciones de violencia de pareja en todos los casos, y así mismo se tuviera la obligación de asistir a tratamiento, ya que estos procesos terapéuticos pueden contribuir en la generación de cambios en las dinámicas familiares a través del aprendizaje de estrategias más adecuadas de manejo de conflictos, así como habilidades personales de los integrantes de la pareja.

Sorprende las decisiones tomadas por los comisarios en relación con las situaciones que desencadenan violencia en las parejas. Como lo muestra la literatura reseñada (Gómez, Ramos \& Rojas, 2010; Medina, 2002; Villamil, 1999), el consumo de alcohol o de sustancias psicoactivas puede constituirse en riesgo de mantenimiento y repetición de la conducta violenta. De acuerdo con lo encontrado, tanto en las pericias psicológicas como lo manifestado en audiencia por las accionantes o por los accionados, en el $36 \%$ de los casos se presentaba consumo de alcohol por parte del accionado y en el $10 \%$ de sustancias psicoactivas.

Se encontró que se ordenó para el accionado el tratamiento específico para el consumo de estas sustancias tan solo en el $9 \%$ de los casos y que, en algunos fallos, aún cuando el operador de justicia consideró este elemento como un factor de riesgo y elemento de intranquilidad para la víctima, no se presentaba una orden específica para el manejo de este factor de manera diferenciada. En cuanto a la orden que el accionado no debe consumir sustancias (alcohol o SPA) en el hogar o ingresar al mismo bajo los efectos de estas, se observa que la proporción es aún menor para el establecimiento de dicha medida, dándose tan solo en un $4 \%$ de todos los casos. Se esperaba que las medidas se hubiesen generado en todos los casos señalados en que se daba la situación de consumo y no en los porcentajes presentes en este estudio. También, llama la atención que, en las pericias psicológicas, se señalara que en el $26 \%$ de los casos, las víctimas reportaron que habían sido intimidadas alguna vez con arma blanca o de fuego y tan solo el $4 \%$ fue amonestado frente a 
esta situación y de este porcentaje, solo el $2 \%$ se había detectado durante la audiencia, por tanto, solo se tuvo en cuenta este aspecto en un $2 \%$ registrado por las pericias psicológicas. La intimidación con armas constituye una forma de violencia emocional, como lo expresó Garzón (2000), con serias consecuencias psicológicas, señaladas por Amor et ál. (2002); Cobo (1999); Jackson, PetriticJackson \& Witte (2002); Pérez (2002) y Radford \& Hester (2006); por tanto, se considera que los operadores de justicia bien podrían suspender al agresor la tenencia, porte y uso de armas, como lo ordena la Ley 1257 de 2008.

Las consecuencias emocionales reseñadas por la literatura científica son coincidentes con las características de la muestra, toda vez que las mujeres participantes del estudio, a quienes se practicó la pericia psicológica, presentaban índices de depresión (74\%); ansiedad (83\%); bajos niveles de autoestima (35\%) y estrés postraumático (39\%). Si bien, es cierto que, dentro de las medidas tomadas se presentó en una alta proporción, la remisión a tratamiento reeducativo y terapéutico a las dos partes (75\%), se considera necesario que se haga énfasis en las remisiones a los aspectos específicos de intervención para la víctima. Teniendo en cuenta que el Plan Obligatorio de Salud de las EPS y del sistema subsidiado de salud no cubre la salud mental de la población, las remisiones a este tipo de tratamientos deberían hacerse a centros especializados de atención psicológica, cuyos costos debería cubrir el agresor, como ordena la Ley 575 del 2000 (Artículo 2) y la Ley 1257 de 2008 (Artículo 1). En esta investigación, tan solo en uno de los fallos de medida de protección definitiva se encuentra la orden de pago, y vale la pena señalar que en este se indica la responsabilidad de asumir los gastos que generen los tratamientos médicos y psicológicos por cada una de las partes, dado que se amonestó a ambos miembros de la pareja.

En cuanto a la decisión de incluir a los hijos dentro de la medida de protección, se encontró que en el $45 \%$ de los casos se tomó esta acción, pero sorprende que esta medida debió tomarse, por lo menos, en el $68 \%$ de los casos, toda vez que así lo mostraron las pericias psicológicas y los interroga- torios en audiencia cuando no se contaba con la pericia psicológica. Esto teniendo en cuenta, como mencionan Atenciano (2009) y Amonacid et ál. (2006), que la violencia intrafamiliar tiene serias consecuencias en la salud mental de los hijos. En esta misma línea de ideas se esperaba encontrar la medida de asistencia a taller o procesos relacionados con aprendizaje de pautas de crianza (otras medidas, Tabla 1) como estrategia para que la modificación de los estilos de crianza y cuidado de los hijos estuviese presente en la misma proporción (66\%) o incluso más de aquella reportada en términos de afectación de los hijos, pero se encontró que se ordenó tan solo en el $23 \%$ de los casos. Se observó que la utilización de la medida de desalojo es poco frecuente, en las medidas de protección analizadas en esta investigación (4\%). Otras de las medidas utilizadas en los fallos de las medidas de protección definitivas analizados con baja utilización (15\%, Tabla 2), se refieren a indicarle al agresor que no puede impedir el ingreso a la vivienda de la víctima, no involucrar a los hijos en los conflictos, asignación de custodia provisional de los hijos a favor de la madre, así como legalizar acuerdos de fijar residencias separadas en tanto que el accionado se retira de la casa, las cuales buscan prevenir la ocurrencia de eventos futuros de violencia. Es posible que esta baja utilización se deba a que los operadores de justicia no tienen en cuenta algunos de los aportes que brindan profesionales de las áreas de psicología y trabajo social, y adicionalmente a que aún no se ha reglamentado la Ley 1257 del 2008.

Por tanto, además de requerir formar a los comisarios en el fortalecimiento de la aplicación de medios probatorios como las pericias psicológicas en sus decisiones, se debe hacer esta labor en el reconocimiento de las implicaciones de la violencia en la salud mental de las víctimas y del grupo familiar en general.

Dentro de las reflexiones necesarias, en cuanto a las decisiones de las CF, es importante analizar cómo algunas regulaciones legales desconocen la complejidad de la situación de violencia intrafamiliar; a modo de ejemplo, en el Acuerdo 155 del 2005 se establece que a través del programa PARVIF "se busca eliminar las causas que la generan" (Artículo 
2) y que esta responsabilidad recae sobre las CF (Alcaldía de Bogotá, 2005). Afirmaciones que parecen olvidar la multicausalidad de esta problemática, donde intervienen factores sociales, económicos, culturales y psicológicos, que se entremezclan y por ende requieren de la intervención de todos los sectores productivos y sociales del Estado y la puesta en marcha de políticas sociales que realmente intervengan las verdaderas causas que generan el malestar en esta sociedad. Si bien es cierto, la existencia de legislación que propende por la protección de los derechos humanos es importante y ha sido un avance en Colombia, es necesario que esté acompañada de acciones macro que modifiquen las verdaderas causas del problema investigado en este estudio, erradicando la pobreza y posibilitando unas relaciones de mayor equidad y justicia.

Una de las conclusiones que se podría plantear, a partir del presente estudio, es que los comisarios de familia, algunas veces, utilizan la pericia psicológica como prueba para demostrar la existencia de violencia, sin embargo, no se observa una asociación para la toma de medidas concretas de protección concordantes con los resultados arrojados por dichas pericias, aún cuando en ocasiones señalan dentro del fallo aspectos específicos de aquellas. Esta tendencia hace pensar que la psicología forense está siendo percibida como una disciplina auxiliar del derecho y no como una disciplina que podría asesorar y orientar en la toma de decisiones importantes dentro de los procesos judiciales. Por tanto, es tarea de los psicólogos jurídicos, en general, y de los forenses, en particular, continuar haciendo investigaciones, publicando avances y abriendo campo en el medio jurídico para posicionar mejor esta disciplina.

Las pericias psicológicas dan cuenta de la posibilidad de ocurrencia de los hechos de violencia, objeto de la medida, además de predecir futuras acciones de acuerdo con las características de los agresores, de la víctima y de la dinámica que se presenta al interior del hogar, lo que puede redundar en brindar elementos adicionales para establecer medidas de protección específicas para cada familia centradas en los aspectos que señala la ley acerca de la prevención de la violencia y estrategias de restablecimiento de derechos de las víctimas, dando cuenta de aspectos específicos y concretos de la intervención psicoterapéutica requerida, así como de aspectos de control-sanción, como el establecimiento de condiciones de utilización de armas, de consumo de alcohol o sustancias psicoactivas, de cuidado de los hijos u otros integrantes familiares, entre otras. Si bien es cierto, como menciona Wrightsman (1999) "cada disciplina se acerca a la construcción del conocimiento y a las normas para la toma de sus decisiones en su propia forma, un abogado y un psicólogo pueden ver el mismo evento desde perspectivas diferentes por su entrenamiento especializado" (p. 221), es de resaltar que allí es donde justamente radica su importancia, dado que es ese entrenamiento diferenciado lo que permite señalar elementos fácticos para la construcción de conocimiento y para una decisión más integral.

Teniendo en cuenta lo señalado se considera fundamental continuar fortaleciendo los procesos de investigación científica, en términos del necesario vínculo entre la academia y la práctica, para generar nuevos conocimientos que fortalezcan los procesos de atención e intervención, especialmente en problemáticas tan complejas como la violencia familiar.

\section{Referencias}

Alcaldía de Bogotá. Acuerdo 155 de 2005 por medio del cual se crea el Programa de seguimiento y apoyo a familias afectadas por la violencia intrafamiliar-PARVIF.

Almonacid, F., Daroch, C. \& Mena, P. (2006). Investigación social sobre violencia conyugal. Red Última Década. Consultado el 29 de octubre de 2010. Recuperado de: http://site.ebrary.com/lib/lablaavirtualsp/ Doc?id=10124092\&ppg=8

Alonso, J. \& Castellanos, J. (2006). Por un enfoque integral de la violencia familiar. Psychosocial Intervention-Intervención Psicosocial, sin mes, 253-274. Recuperado de: http://redalyc.uaemex.mx 
Amor, P., Echeburúa, E., De Corral, P., Zubizarreta, I. \& Sarasua, B. (2002). Repercusiones psicopatológicas de la violencia doméstica en la mujer en función de las circunstancias del maltrato. International Journal of Clinical and Health Psychology, mayo, 227-246. Recuperado de: http://redalyc.uaemex.mx

Arenas, J. (2003). Pruebas penales (2 ${ }^{\mathrm{a}}$. ed.). Bogotá: Ediciones Doctrina y ley.

Atenciano, B. (2009). Menores Expuestos a Violencia contra la Pareja: Notas para una Práctica Clínica Basada en la Evidencia. Clínica y Salud, 20(3), 261-272.

Cáceres, J. (2007). Violencia doméstica: lo que revela la investigación básica con parejas. Papeles del Psicólogo, enero-abril, 57-62. Recuperado de: http://redalyc.uaemex.mx

Cobo, J. (1999). Manual de actuación sanitaria, policial, legal y social frente a la violencia doméstica. Barcelona: Ed. Masson.

Código de la Infancia y la Adolescencia (2006). Ley 1098 de 2006. Bogotá: Oficina de Comunicaciones ICBF.

Código de Procedimiento Civil. Decretos 1400 y 2919 de 1970, por los cuales se expide el Código de Procedimiento Civil. Modificado por el Decreto 3930 de 2008, por el Decreto 21 de 2009, por la Ley 794 de 2003, por la Ley 572 de 2000, por la Ley 388 de 1997, por el Decreto 2282 de 1989, por el Decreto 522 de 1988 , y por la Ley 2 de 1984 y por la Ley 1194 de 2008.

Consejería Presidencial para la Política Social. (2001). Reflexiones para la Intervención en la Problemática Familiar. Módulo IV: Violencia Intrafamiliar. Bogotá: Consejería Presidencial para la Política Social/ PNUD.

Corsi, J., Domen, M. , \& Sotés, M. (1995). Violencia masculina en la pareja: una aproximación al diagnóstico y a los modelos de intervención. Buenos Aires: Paidós.

Dutton, D. \& Golant, S. (1997). El golpeador: un perfil psicológico. Buenos Aires: Paidós.

Echeburúa, E. (1994). Personalidades violentas. Madrid: Pirámide.

Echeburúa, E. \& De Corral, P. (1998). Manual de violencia familiar. Madrid: Siglo XXI.

Echeburúa, E., De Corral, P., Sarasua, B. \& Zubizarreta, I. (1998). Mujeres víctimas de maltrato. En E. Echuburúa \& P. De Corral (Coords.). Manual de violencia familiar (969). Madrid: Siglo XX.

Esbec, E. \& Gómez-Jarabo, G. (1999). Violencia y delincuencia: una visión psicopatológica y psicosocial. Psicopatología, 19, 51-57.

Garzón, R. (2000). El peritaje sociofamiliar frente a la violencia conyugal. Cali: Publicaciones Universidad Libre.

Gómez, G., Ramos, A., \& Rojas, R. (2010). Adaptación de la versión española del S.A.RA., Manual para la valoración del riesgo de violencia contra la pareja al contexto colombiano. Tesis de Maestría no publicada, Bogotá: Universidad Santo Tomás.

Instituto Nacional de Medicina Legal y Ciencias Forenses. (2001). Guía práctica para el dictamen de lesiones personales. Bogotá: INMLCF.

Instituto Nacional de Medicina Legal y Ciencias Forenses. (2006, 2007, 2008, 2009). Forensis: Datos para la Vida. Bogotá: INMLCF.

Jackson, T., Petritic-Jackson, P. \& Witte, T. (2002). Mental Health Assessment Tools and Techniques for Working With Battered Women. En A. Roberts \& A. Roberts (Ed.). Handbook of Domestic Violence Intervention Strategies: Policies, Programs, and Legal Remedies (278-308). USA: Oxford University 
Press. Consultado el 27 de marzo de 2010. Recuperado de: http://site.ebrary.com/lib/ lablaavirtual/Doc?id=10085252

Labrador, F., Rincón, P., De Luis, P. \& FernándezVelasco, R. (2004). Mujeres víctimas de la violencia doméstica. Programa de actuación. Madrid: Pirámide.

Ley 294 de 1996. Normas para prevenir, remediar y sancionar la violencia intrafamiliar, Julio de 1996.

Ley 575 de febrero de 2000 por medio de la cual se reforma parcialmente la Ley 294 de 1996.

Ley 1257 de diciembre de 2008. Por la cual se dictan normas de sensibilización, prevención y sanción de formas de violencia y discriminación contra las mujeres, se reforman los Códigos Penal, de Procedimiento Penal, la Ley 294 de 1996 y se dictan otras disposiciones.

Medina, J. (2002). Violencia contra la mujer en la pareja: investigación comparada y situación en España. Valencia: Tirant lo Blanch.

Organización Mundial de la Salud. (2009). Violencia contra la mujer. Consultado el 1 de marzo de 2010. Recuperado de: http:// www.who.int/mediacentre/factsheets/ fs239/es/index.html

Ospino, J. A. (2004). Metodología de la investigación en ciencias de la salud. Bogotá: Editorial Universidad Cooperativa de Colombia.

Pérez, A. (2002). Psicopatología de la mujer víctima de violencia doméstica. En Tercer Congreso Virtual de Psiquiatría Interpsiquis 2002. psiquiatría.com. Consultado el 22 enero 2010. Recuperado de: http://www.psiquiatria.com/ psiquiatria/revista/69/4840/?++interactivo

Pérez, E., Lledias, E., \& Giraldo, A. (2008). Convivencia familiar: una lectura aproximativa desde elementos de la Psicología Social.
Diversitas: Perspectivas en Psicología, 4(2), 427-441.

Procuraduría General de la Nación (2009). Aproximación a la situación de violencia contra las mujeres en Colombia: informe de vigilancia. En Procurando Equidad N.4: Vigilancia superior a la garantía de los derechos desde la perspectiva de género. Bogotá: Procuraduría General de la Nación.

Profamilia (2005). Salud Sexual y Reproductiva en Colombia. Encuesta Nacional de Demografía y Salud ENDS. Consultado el 20 de septiembre de 2009. Recuperado de: http://www. profamilia.org.co/encuestas/02consulta/13 violencia/02fisica.htm

Radford, L. \& Hester, M. (2006). Mothering Through Domestic Violence. London, GBR: Jessica Kingsley Publishers. Consultado el 27 de marzo de 2010. Recuperado de: http://site.ebrary.com/lib/lablaavirtual/ Doc?id $=10156028$

Ravazzola, M. (1999). Historias infames: los maltratos en las relaciones. Buenos Aires: Paidós.

Rodríguez, L., Padilla, A., Rodríguez, L. \& Díaz, F. (2010). Análisis de la justicia restaurativa para atender casos de violencia intrafamiliar en el Centro de Atención Integral a Víctimas de Violencia Intrafamiliar (CAVIF) de la Fiscalía General de la Nación, Colombia. Diversitas: Perspectivas en Psicología, 6(2), 355-373. Recuperado de: http://www.usta. edu.co/otras_pag/revistas/diversitas/revistas.html

Rosen, K., Stith, M. \& Williams, M. (1992). Psicosociología de la violencia en el hogar. Bilbao: Desclée de Brouwer.

Secretaría de Gobierno de Bogotá. (2003). Mossavi. Modelo de estrés social para la prevención y atención de la violencia intrafamiliar. Manual para docentes. Bogotá: Gamma-Idear. 
Secretaría Distrital de Integración Social. (2007a). Boletín Violencia Intrafamiliar en Bogotá D.C. Años 2004, 2005 y 2006. Bogotá: Editorial Visuales Dar Ltda.

Secretaría Distrital de Integración Social. (2007b). Plan distrital para la prevención de la violencia intrafamiliar, el maltrato infantil, la violencia y explotación sexual. Bogotá: Anthropos.

Sarasua, B., Zubizarreta, I., Echeburúa, E. \& De Corral, P. (2007). Perfil psicopatológico dife- rencial de las víctimas de violencia de pareja en función de la edad. Psicothema, 459-466. Recuperado de: http://redalyc.uaemex.mx

Villamil, E. (1999). Protección familiar: visión constitucional. Bogotá: Doctrina y Ley.

Walker, L. (2000). The Battered Woman Syndrome (2da Ed.). New York: Springer.

Wrightsman, L. (1999). Judicial decision making: is psychology relevant? New York: Kluwer. 